\title{
Non-extensivity in magnetic systems: possible impact on Mossbauer results
}

\author{
Ashok Razdan \\ Astrophysical Sciences Division \\ Bhabha Atomic Research Centre \\ Trombay, Mumbai- 400085 \\ email:akrazdan@barc.gov.in \\ Tel: +9122 25591798; fax: +912225505151 \\ PACS:71.70.Ej,75.47.Lx,76.80.+y,63.70.+h
}

Keywords: Non-extensive, manganites, energy positions, Zeeman splitting

\begin{abstract}
:
Energy positions of pure magnetic transitions in Mossbauer Spectroscopy are calculated using non-extensive approach. It is observed that these new calculated energy positions so obtained, may have strong overlap with those energy positions obtained from combined effect of magnetic and quadrupole interactions using standard statistical physics.
\end{abstract}

\section{Motivation:}

Non-extensive statistics is being increasingly used to explain anomalous results observed in various physical systems like turbulence in plasma,Cosmic ray background radiation, self gravitating systems, econo-physics,electron positron annihilation, chaos, linear response theory, Levy type anomalous super diffusion, Lamb Mossbauer factor,specific heat in glasses ,low dimensional systems [1-9] etc. The non-extensive approach is based on non-extensive entropy which is given as

$$
S_{q}=\frac{1-\sum p_{i}^{q}}{q-1}
$$

$p_{i}$ are the probabilities of the microscopic states with $\sum p_{i}=1$. In the limit of $\mathrm{q}$ 
$\rightarrow 1, S_{1}=-\sum p_{i} \ln \left(p_{i}\right)$ which is Boltzmann-Gibbs-Shannon entropy. It has been shown that non-extensive features get manifested in those systems which have long range forces, long memory effects,inhomogeneous systems or in those systems which evolve in (non Euclidean like space-time) fractal space time [10 and reference therein]. It has been shown in recent times that non-extensivity is also relevant to magnetic systems. Using inputs from various experimental studies, manganites have been identified as possible non-extensive objects. Manganites have long range Coulomb interactions[11,12,13], fractal like clusters [14,15] and intrinsic inhomogeneity $[16,17,18]$ It has been further shown that non-extensivity parameter ' $\mathrm{q}$ ' is a measure of inhomogeneity . Correct predictions about bulk magnetization have been made using non-extensive approach $[19,20,21]$. Nonextensivity in griffths phase has also been investigated [22,23]. This relevance of non-extensivity in magnetism [24] has motivated us to explore its impact on Mossbauer parameters of magnetic systems.

\section{Nuclear Zeeman splitting:}

Magnetic hyperfine splitting arises from interaction between magnetic moments of the ground and excited states of nucleus [25] with the internal or external magnetic field. The interaction Hamiltonian is given as

$$
H=-\mu \cdot H_{n}
$$

where $\mu$ is magnetic moment and $H_{n}$ is the magnetic field. This interaction completely lifts the degeneracy of nuclear levels of spin I and energy $E_{0}$ is split into $(2 \mathrm{I}+1)$ levels. The energy levels obtained are

$$
E_{m}=E_{0}-g_{n} \mu_{n} H_{n} m_{I}
$$

where $m_{I}=\mathrm{I}, \mathrm{I}+1, \ldots-\mathrm{I}, g_{n}$ is the splitting factor ( gryomagnetic ratio), $\mu_{n}$ is the nuclear magneton. The magnetic interaction splits both the ground and excited states between which transitions take place. Thus in ${ }^{57} \mathrm{Fe}$ six transitions are obtained and the transition probabilities are given by well know Clebsch-Gordon coefficients. The transitions probabilities, their angular variations and energy positions are given in reference [25]. 
For $\pm \frac{3}{2} \rightarrow \pm \frac{1}{2}$ transition, the energy positions of Ist and 6th transition corresponds to [25].

$$
\text { Energy }-\operatorname{Position}(1,6)=\left(E_{0} \pm \frac{3}{2} g_{e} \mu_{n} H_{n} \pm \frac{1}{2} g_{g} \mu_{n} H_{n}\right)
$$

where $g_{e}$ and $g_{g}$ are the ratios of the nuclear magnetic moment to the nuclear magneton $\mu_{n}$ for the ground and the excited states respectively.

\section{Non-extensive approach in magnetic splitting:}

For non-extensive case, $[19,20,21,22]$ interaction Hamiltonian is given by

$$
H=-\mu_{n e} \cdot H_{n}
$$

where $\mu_{n e}$ is non-extensive magnetic moment. The expression for non-extensive magnetization is given as

$$
M_{q}=\frac{\mu_{n e}}{(2-q)}\left[\operatorname{coth}_{q}(x)-\frac{1}{x}\right]
$$

where $\mathrm{x}=\frac{\mu_{n e} H}{k T}$. It has been found that relationship

$$
\mu_{n e}=(2-q) \mu
$$

hold for $0 \leq q \leq 1$. This relationship has also been derived using [21] generalized Brillouin function. To obtain energy position for the non-extensive case, replace $\mu_{n}$ in equation (4) by $\mu_{n e}$ and use equation (7).Thus for $\pm \frac{3}{2} \rightarrow \pm \frac{1}{2}$ transition of ${ }^{57} \mathrm{Fe}$ nucleus, non-extensive energy positions of Ist and 6th transition correspond to

$$
\text { Energy }-\operatorname{Position}(1,6)=\left(E_{0} \pm \frac{3}{2}(2-q) g_{e} \mu_{n} H_{n} \pm \frac{1}{2}(2-q) g_{g} \mu_{n} H_{n}\right)
$$

Similarly for $\pm \frac{1}{2} \rightarrow \pm \frac{1}{2}$ transition of ${ }^{57} \mathrm{Fe}$ nucleus , non-extensive energy positions of 2 nd and 5 th transition correspond to

$$
\text { Energy }-\operatorname{Position}(2,5)=\left(E_{0} \pm \frac{1}{2}(2-q) g_{e} \mu_{n} H_{n} \pm \frac{1}{2}(2-q) g_{g} \mu_{n} H_{n}\right)
$$

For $\mp \frac{1}{2} \rightarrow \pm \frac{1}{2}$ transition of ${ }^{57} \mathrm{Fe}$ nucleus, non-extensive energy positions of 3rd and 4th transition correspond to

$$
\text { Energy }-\operatorname{Position}(3,4)=\left(E_{0} \mp \frac{1}{2}(2-q) g_{e} \mu_{n} H_{n} \pm \frac{1}{2}(2-q) g_{g} \mu_{n} H_{n}\right) \text {. }
$$




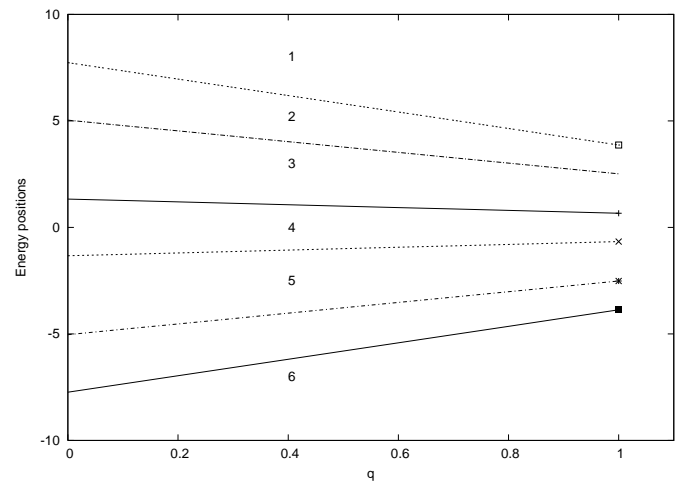

Figure 1: Energy postion v/s q parameter for pure Mossbauer magnetic transitions

Each energy position in equation (7-9) corresponds to two energies. Thus the relationship between non-extensive and standard energy position can be written as

$$
(\text { Energy }- \text { Position })_{\text {non-extensive }} \propto(2-q)(\text { Energy }- \text { Position })_{\text {standard }}
$$

All the new energy positions obtained from non-extensive approach will have $\mathrm{q}$ dependence.

Equations (8-10) have been obtained using non-extensive approach. In the limit of $\mathrm{q} \rightarrow 1$, non-extensive approach reduces to standard Boltzmann-Gibbs approach (hereafter referred as standard case in the text).

\section{Results and Discussion:}

Energy position dependence on q parameter has been plotted in figure 1. For $q=1$ the standard case, the energy positions correspond to well known positions of normal magnetic splitting. These positions in figure 1 are represented by various symbols like open/close square, cross, plus etc. in the figure. For all other values of q the energy positions in the figure 1 corresponds to non-extensive nature. In figure 1 curves ' 1 ' and ' 6 ' correspond to the $\pm \frac{3}{2} \rightarrow \pm \frac{1}{2}$ transition, curves '2' and ' 5 ' corresponds to $\pm \frac{1}{2} \rightarrow \pm \frac{1}{2}$ transition and curves ' 3 ' and ' 4 ' corresponds to $\mp \frac{1}{2} \rightarrow \pm \frac{1}{2}$ transition respectively.

For pure magnetic splitting in the standard case the positions of energies are 
fixed. But combined effect of magnetic and quadrupole interaction ( for standard case) results in shifting of these energy positions. The magnetic energy levels $[25,26]$ for the standard case obtained when treating the quadrupole interaction as a perturbation is given as

$$
E=-g_{n} \mu_{n} H_{n} m_{I}+\left((-1)^{m_{I}+\frac{1}{2}}\right) \frac{e^{2} V_{z z} Q}{4}\left(\frac{3 \cos ^{2} \theta-1}{2}\right)
$$

where $\mathrm{Ze}$ is the nuclear charge, eQ is the quadrupole moment and $V_{z z}$ is the electric field gradient along $\mathrm{z}$ axis. The above equation holds for $e^{2} V_{z z} Q<<$ $\mu_{n} H_{n}$ i.e. quadrupole interaction is much less than the magnetic interaction. The combined effect of magnetic and quadrupole interaction in the standard case [25] is parameterized by $\lambda$ which is defined as

$$
\lambda=\frac{\frac{e^{2} V_{z z} Q}{2 I(2 I-1)}}{\frac{\mu_{n} H_{n}}{I}}
$$

The value of $\lambda$ is between 0 to 1 . In the figure 2 we have plotted energy position dependence on $\lambda$. The curves 1 and 2 correspond to $\frac{3}{2} \rightarrow \frac{1}{2}$ transition. The curves 3 and 4 correspond to $\frac{1}{2} \rightarrow \frac{1}{2}$ transition and curves 5 and 6 correspond to $\frac{1}{2} \rightarrow \frac{1}{2}$ transition respectively. These curves in figure 2 have been obtained for $\theta=0$ degree (curves 2,4 and 6) and $\theta=90$ degrees. (curves 1,3 and 5) respectively.

For standard case, the presence of quadrupole interaction affects magnetic transitions in such a way that energy positions are shifted to any position in figure 2 for a given value of $\lambda$. However,it is clear from figure 1 that in the nonextensive case, the energy positions are shifted even for pure magnetic splitting for a given value of $q$. Comparing figures 1 and 2 , it is obvious that some of the values may overlap or some curves may even cross each other, in which case a non-extensive pure magnetic transition may have same energy position as standard mixed transition case.

Large number of Mossbauer results for magnetism [26-29] in general and manganites in particular have been produced in which presence of quadrupole interaction have been assumed to explain the shift in energy positions of various transitions. If manganites /magnetic systems have non-extensive nature 


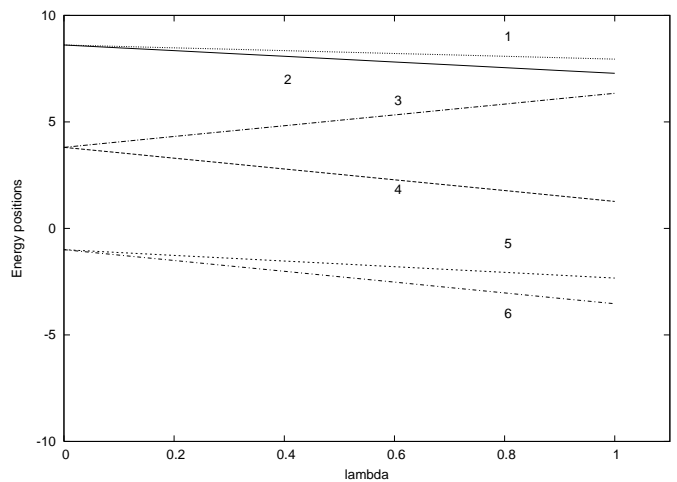

Figure 2: Energy Position of Mixed transitions v/s $\lambda$ plot for angle 0 and 90.

than energy positions will indeed shift, yet transitions may be pure magnetic in nature. This means all the calculations of assuming mixed transitions for manganites/magnetic systems may be wrong and need to be reinvestigated.

Thus it is suggested that for magnetic systems in general and manganites in particular inverse susceptibility experiments [22] may be used first to establish if the system (sample) is non-extensive or normal. For normal systems standard protocol of Mossbauer Spectroscopy should be followed. For non-extensve systems, using the value of q (obtained from inverse susceptibility experiments) correction should first be introduced on the energy positions so that non-extensive energy positions are renormalized to normal system values. After introducing non-extensive corrections, standard Mossbauer protocol of calculating hyperfine parameters may be followed.

\section{References:}

1. B.M.Boghosian, Phys. Rev. E 53(1996)4745

2. C.Tsallis, F.C. Sa Barreto and E.D.Loh, Phys. Rev. E 52(1995) 1447

3. V.H.Hamity and D.E.Barraco, Phys. Rev. Lett. 76(1996)4664

4. I.Bediaga, E.M.F.Curado and J. Miranda, Physica A 286(2000)156

5. C.Tsallis, A.R.Plastino and W. -M.Zheng, Choas,Solitons and Fractals 
8(1997)885,

Y.Weinstein, S.Lloyd and C.Tsallis, Phys. Rev. Lett. 89(2002)214101

6. A.Razdan, Phys. Lett. A 321(2004)190

7. A.Razdan, Phys. Lett. A 341(2005)504

8. C.Tsallis, S.V.F. Levy, A.M.C.Souza and R.Maynard, Phys. Rev. Lett. $75(1995) 3589$

9. I.Koponen, Phys. Rev. E 55(1997)7759

10. M.L.Lyra and C.Tsallis, Phys. Rev. Lett. 80(1998)53

11. C.Tsallis, Physica A 221(1995)277-290, C.Tsallis, R.S.Mendes, A.R.Plastino, Physica A 261(1998)534

12. J.Lorenzana et. al., Phys. Rev. B 64(2001)235127

13. J.Lorenzana et. al., Phys. Rev. B 64(2001)235128

14. A.Moreo et.al., Science 283(1999)2034

15. E.Dagotto et. al, Phys. Rep. 344(2001)1

16. M.Ausloos et. al, Phys. Rev.B 66(2002)174436

17. E.Dagotto, Nanscale Phase Separation and Clossal Magnetoresistance, Springer-Veralg,Heidelberg 2003

18. T.Becker et. al., Phys. Rev. Lett. 89(2002)237203

19. S.Kumar and P.Majumdar, Phys. Rev.Lett. 92(2004)126602

20. K.H.Ahn et. al., Nature (London) 428(2004)402

21. M.S.Reis et. al., Europhys.Lett. 58(2002)42

22. M.S.Reis et. al., Phys. Rev. B 66(2002)134417

23. M.S.Reis et. al., Phys. Rev. B 68(2003)014404 
24. M.S.Reis et. al., Phys. Rev. B73(2006)092401

25. V.G.Bhide, Mossbauer Effect and its Applications, TATA McGraw Hill Publishing, New Delhi 1973

26. R.S.Preston et. al., Phys. Rev. 128(1962)2207

27. J.L.Dormann and D.Fiorani(editors), Manetic Properties of Fine Particles , North Holland 1992

28. B.V.Thosar et. al. (editors), Advances in Mossbauer Spectroscopy, Elsevier Publishers 1983

29. R.L.Cohen, Elements of Mossbauer Spectroscopy, Academic Press 1976 